\title{
Krull dimension and integrality of symmetric algebras
}

\author{
Aron Simis* and Wolmer V. Vasconcelos ${ }^{\dagger}$
}

For a finitely generated module $E$ over the Noetherian ring $R$ we consider formulas for the Krull dimension of the symmetric algebra $S(E)$. A result of Huneke and Rossi is re-proved and an efffective formula is derived that reads the dimension of $S(E)$ from a presentation of $E$. They provide a first line of obstructions for $S(E)$ to be an integral domain. For algebras of codimension at most four we give methods, including computer-assisted ones, to ascertain whether $S(E)$ is a Cohen-Macaulay domain.

\section{Introduction}

Let $R$ be a Noetherian ring and $E$ a finitely generated $R$-module. If $E$ is given by the presentation

$$
R^{m} \stackrel{\varphi}{\longrightarrow} R^{n} \longrightarrow E \longrightarrow 0, \varphi=\left(a_{i j}\right)
$$

its symmetric algebra is the quotient of the polynomial ring $R\left[T_{1}, \cdots, T_{n}\right]$ by the ideal $J(E)$ generated by the 1 -forms

$$
f_{j}=a_{1 j} T_{1}+\cdots+a_{n j} T_{n}, j=1, \ldots, m .
$$

Two main themes on the ideal theory of $S(E)$ are discussed here: Explicit formulas for the Krull dimension of $S(E)$ and the exploration of properties of the module $E$ that are mirrored on $S(E)$.

AMS 1980 Mathematics Subject Classification (1985 Revision). Primary 13H10; Secondary 13-04, 13C05, $13 \mathrm{C} 15$

-Partially supported by CNPq, Brazil

'Partially supported by the NSF 
It was only recently that Huneke and Rossi, cf. [8], linked the dimension of $S(E)$ to a number introduced by Forster (cf. [3]) that bounds the number of generators of $E$ as expressed in its local data. It made the search for dimension formulas for $S(E)$ much easier. Based on slightly different ideas we give another proof of that result, and while in [13] we only estimated the Krull dimension of $S(E)$ in terms of the heights of the Fitting ideals of $E$, here we use [8] to give the exact formula. It makes for an effective way of determining the Krull dimension of $S(E)$.

With regard to the other topic, we focus on the question: If $E$ is a torsion-free module, when is $S(E)$ an integral domain, integrally closed, or Cohen-Macaulay? If $R$ is a polynomial ring over a prime field, some of these issues can be approached through symbolic computation packages. It is nonetheless interesting to develop a horizon of the likely answers.

Our examination is focused on modules of projective dimension at least two and rank greater than one, the rank one case being extensively looked at in [4]. The main results are descriptions of integral domains, particularly in codimension at most four. It emphasizes the steps that may be taken to show symmetric algebras to be Cohen-Macaulay integral domains.

\section{Krull dimension of symmetric algebras}

To give the proof of the dimension theorem of [8] we need a few preliminary observations about dimension formulas for graded rings. $M$. Kühl has, independently, noticed even broader formulas.

The derivation of the dimension formula in terms of the Fitting ideals of $E$ seems to require that $R$ be catenarian. This is somewhat unsatisfactory since one of our motivations was to remove entirely this condition from [8].

\subsection{Dimension formulas}

For a prime ideal $P$ of $R$ denote by $v\left(E_{P}\right)$ the minimal number of generators of the localization of $E$ at $P$. It is the same as the torsion free rank of the module $E / P E$ over the ring $R / P$. The Forster number of $E$ is:

$$
b(E)=\sup _{P \in S p e c(R)}\left\{\operatorname{dim}(R / P)+v\left(E_{P}\right)\right\} .
$$

Theorem 1.1.1 (Huneke-Rossi) $\operatorname{dim} S(E)=b(E)$.

The following seems to have gone unnoticed: 
Lemma 1.1.2 Let $B$ be a Noetherian integral domain that is finitely generated over a subring $A$. Suppose there exists a prime ideal $Q$ of $B$ such that $B=A+Q, A \cap Q=O$. Then

$$
\operatorname{dim}(B)=\operatorname{dim}(A)+\operatorname{height}(Q)=\operatorname{dim}(A)+\operatorname{tr} \cdot \operatorname{deg} \cdot{ }_{A}(B) .
$$

Proof. We may assume that $\operatorname{dim} A$ is finite; $\operatorname{dim}(B) \geq \operatorname{dim}(A)+$ height $(Q)$ by our assumption. On the other hand, by the standard dimension formula of [10], for any prime ideal $P$ of $B, \mathbf{p}=P \cap A$, we have

$$
\text { height }(P) \leq \operatorname{height}(\mathbf{p})+\operatorname{tr} \cdot \operatorname{deg} \cdot A(B)-\operatorname{tr} \cdot \operatorname{deg} \cdot k(\mathbf{p}) k(P) .
$$

The inequality $\operatorname{dim}(B) \leq \operatorname{dim}(A)+\operatorname{height}(Q)$ follows from this formula and reduction to the affine algebra obtained by localizing $B$ at the zero ideal of A.

There are two cases of interest here. If $B$ is a Noetherian graded ring and $\mathrm{A}$ denotes its degree 0 component then:

$$
\operatorname{dim}(B / P)=\operatorname{dim}(A / \mathbf{p})+\operatorname{tr} \cdot \operatorname{deg} \cdot k(\mathbf{p}) k(P)
$$

and

$$
\operatorname{dim}\left(B_{\mathbf{p}}\right)=\operatorname{dim}\left(A_{\mathbf{p}}\right)+\operatorname{tr} \cdot \operatorname{deg} \cdot A(B) .
$$

Proof of the theorem. We make use of the observation that if $R$ is an integral domain, then the $R$-torsion submodule of a symmetric algebra $S(E)$ is a prime ideal of $S(E)$. of

Let $\mathbf{p}$ be a prime ideal of $R$; denote by $T(\mathbf{p})$ the $R / \mathbf{p}$-torsion submodule

$$
S(E) \otimes R / \mathbf{p}=S_{R / \mathbf{p}}(E / \mathbf{p} E) .
$$

By the formula above we have

$$
\begin{aligned}
& \operatorname{dim} S(E) / T(\mathbf{p})=\operatorname{dim}(R / \mathbf{p})+\operatorname{tr} \cdot \operatorname{deg}_{\cdot R / \mathbf{p}} S(E) / T(\mathbf{p}) \\
& =\operatorname{dim}(R / \mathbf{p})+v\left(E_{\mathbf{p}}\right)
\end{aligned}
$$

and it follows that $\operatorname{dim} S(E) \geq b(E)$.

Conversely, let $P$ be a prime of $S(E)$ and put $\mathbf{p}=P \cap R$. It is clear that $T(\mathbf{p}) \subset P$,

$$
\operatorname{dim} S(E) / P \leq \operatorname{dim} S(E) / T(\mathbf{p}),
$$

and $\operatorname{dim} S(E) \leq b(E)$ as desired. 
We are now going to translate this formula into an effective formula for computing the dimension of $S(E)$ in terms of the Fitting ideals of $E$ (see also [4, Lemma 8.2]).

Let $R$ be a Noetherian domain and let

$$
\varphi: R^{m} \longrightarrow R^{n}
$$

be a presentation of the $R$-module $E$. We intend to express $\operatorname{dim} S(E)$ in terms of the sizes of the determinantal ideals of the matrix $\varphi$.

For each integer $t \geq 1$ denote by $I_{t}(\varphi)$ the ideal generated by the $t \times t$ minors of $\varphi$. Consider the condition $\left({ }^{*}\right)$ :

$$
\operatorname{height}\left(I_{t}(\varphi)\right) \geq \operatorname{rank}(\varphi)-t+1, \quad 1 \leq t \leq \operatorname{rank}(\varphi) .
$$

A lower bound for $b(E)$ is $b_{0}(E)=\operatorname{dim} R+\operatorname{rank}(E)$, the value corresponding to the generic prime ideal in the definition of $b(E)$. We show that the correction from $b_{0}(E)$ can be explained by how deeply the condition $\left(^{*}\right)$ is violated. Set $m_{0}=\operatorname{rank}(\varphi)$, so that $\operatorname{rank}(E)=n-m_{0}$. Without loss of generality we assume that $R$ is a local $\operatorname{ring}, \operatorname{dim} R=d$. Consider the descending chain of affine closed sets:

$$
V\left(I_{m_{0}}(\varphi)\right) \supseteq \cdots \supseteq V\left(I_{1}(\varphi)\right) \supseteq V(1) .
$$

Let $P \in \operatorname{Spec}(R) ;$ if $I_{m_{0}}(\varphi) \nsubseteq P, \operatorname{rank}(E / P E)=n-m_{0}, \operatorname{dim}(R / P)+$ $\operatorname{rank}(E / P E) \leq b_{0}(E)$. On the other hand, if $P \in V\left(I_{t}(\varphi)\right) \backslash V\left(I_{t-1}(\varphi)\right)$, we have $\operatorname{rank}(E / P E)=n-t+1$; if $\left(^{*}\right)$ is not violated at $t$, the height of $P$ is at least $m_{0}-t+1$ and again $\operatorname{dim}(R / P)+\operatorname{rank}(E / P E) \leq b_{0}(E)$.

Define the following measure on $[1, \operatorname{rank}(\varphi)]$ :

$$
d(t)= \begin{cases}m_{0}-t+1-\text { height }\left(I_{t}(\varphi)\right) & \text { if }\left(^{*}\right) \text { is violated at } t \\ 0 & \text { otherwise. }\end{cases}
$$

Finally, if we put $d(E)=\sup _{t}\{d(t)\}$, we have the following dimension formula.

Theorem 1.1.3 Let $R$ be an equi-dimensional catenarian domain and let $E$ be a finitely generated $R$-module. Then $b(E)=b_{0}(E)+d(E)$.

Proof. Assume that $\left({ }^{*}\right)$ fails at $t$, and let $\boldsymbol{P}$ be a prime as above. We have

$$
\operatorname{height}(P) \geq m_{0}-t+1-d(t),
$$


and thus

$\operatorname{dim}(R / P)+\operatorname{rank}(E / P E) \leq\left(d-\left(m_{0}-t+1-d(t)\right)\right)+(n-t+1)=b_{0}(E)+d(t)$.

Conversely, pick $t$ to be the least integer where the largest deficit $d(t)$ occurs. Let $P$ be a prime ideal minimal over $I_{t}(\varphi)$ of height exactly $m_{0}-t+$ $1-d(t)$. From the choice of $t$ it follows that $P \notin V\left(I_{t-1}(\varphi)\right)$, as otherwise the deficit at $t-1$ would be even higher. Since $R$ is catenarian the last displayed expression gives the desired equality.

Under the same hypotheses on $R$ and $E$ (see also [5, Proposition 8.4]):

Corollary 1.1.4 If $S(E)$ is unmixed then $\operatorname{dim} S(E)=\operatorname{dim} R+\operatorname{rank}(E)$.

The following is a slight generalization of [13, Corollary 2.2]:

Corollary 1.1.5 Let $s$ be the number of elements in the largest subinterval of $[1, \operatorname{rank}(\varphi)]$ where $(*)$ is violated. Then $\operatorname{dim} S(E) \leq b_{0}(E)+s$.

Example 1.1.6 Here is a simple illustration: If $E=\operatorname{coker}(\varphi)$

$$
\varphi=\left[\begin{array}{lll}
0 & x & x \\
y & z & 0 \\
x & 0 & x
\end{array}\right],
$$

$m_{0}=3, d(1)=d(3)=0$, but $d(2)=1$, so $\operatorname{dim} S(E)=3+1$.

Example 1.1.7 Let $X=\left(x_{i j}\right)$ a generic, symmetric $n \times n$ matrix, $n \geq 3$. Denote by $R$ the polynomial ring $k\left[x_{i j}\right]$, and let $I$ be the ideal generated by the minors of order $n-1$. That $d(I)=0$ can be verified from [9], and therefore $\operatorname{dim} S(I)=\operatorname{dim} R+1$.

Example 1.1.8 We shall now mention an example in reverse. For a positive integer $n$, let $X=\left(x_{i j}\right)$ and $Y=\left(y_{i j}\right)$ be square $n \times n$ matrices in $2 n^{2}$ indeterminates.

The ideal generated by the entries of

$$
[X, Y]=X \cdot Y-Y \cdot X,
$$

defines the variety of commuting pairs of $n \times n$ matrices. If we fix the $x_{i j}$ variables, it may be looked at as the equations for the symmetric algebra of 
an $R=\mathbf{C}\left[\boldsymbol{x}_{i j}^{\prime} s\right]$-module $E_{n}$. That is, $E_{n}$ is defined by the Jacobian matrix of this ideal with respect to the $y_{i j}$-variables. It is not difficult to show that $E_{n}$ has rank $n$ in all cases and, in view of the fact (cf. [6], [11]) that $S\left(E_{n}\right)$ has dimension $n^{2}+n$, it follows, by 1.1 .3 , that $E_{n}$ satisfies the condition (*) of section 2.

For $n=3, E_{3}$ has projective dimension two and its symmetric algebra is a Cohen-Macaulay integral domain. This was proved using Macaulay, as reported variously. In the general case, we expect its projective dimension to be still two and $S\left(E_{n}\right)$ to be an integral domain.

\subsection{Ideals of linear type}

We shall now connect Theorem 1.1.1 to an abstract dimension formula. First a definition is recalled. For an ideal $I$ of a commutative ring $R$ there exists a canonical mapping

$$
\alpha: S(I) \longrightarrow \mathcal{R}(I)
$$

from the symmetric algebra of $I$ onto its Rees algebra. $I$ is said to be of linear type if $\alpha$ is an isomorphism.

Theorem 1.2.1 Let $R$ be a Noetherian ring and let $I$ be an ideal of linear type contained in the Jacobson radical of $R$. Then

$$
\operatorname{dim}(R)=\sup _{P \supseteq \underline{I} I}\left\{\operatorname{dim}(R / P)+v\left(I_{P}\right)\right\} .
$$

Proof. Since $I$ is of linear type, $S_{R / I}\left(I / I^{2}\right)=g r_{I}(R)$. On the other hand, $\operatorname{dim} g r_{I}(R)=\operatorname{dim} R$, cf. [10, page 122]. Applying Theorem 1.1.1 one obtains the assertion.

As it will be seen, Theorem 1.1.1 is, in turn, a consequence of this formula. The interplay provided by this equivalence is rewarding; for instance, it provides for a very short proof of Theorem 1.1.1 when $R$ is catenarian.

Given an $R$-module $E$, the irrelevant ideal $I=S(E)_{+}$of the symmetric algebra $B=S(E)$ is of linear type, cf. [4, page 87]. Note that

$$
S(E)=S_{B / I}\left(I / I^{2}\right)
$$

so that if we localize $B$ at the multiplicative set $1+I$ we ensure all the hypotheses of the theorem above. What remains is to observe that each prime ideal of $S(E)$ that contains $S(E)_{+}$has the form $\mathbf{p}+S(E)_{+}$, where $\mathbf{p}$ is a prime ideal of $R$.

There are many known classes of ideals of linear types. For the ideals of Example 1.1.7 the answer is not known in full $(n \geq 4)$. 


\section{Integral domains}

The conditions under which the symmetric algebra $S(E)$ is an integral domain have been a source of interest. The situation is well understood for modules of projective dimension one (cf. $[1],[7],[13]$ ), for several classes of ideals (see [4] for a survey) and have been an incentive to the development of several generalizations of the notion of regular sequences and the theory of the approximation complexes. Here we discuss this question with regard to modules of projective dimension two.

Throughout this section $R$ will be a Cohen-Macaulay integral domain, although the full strength of this condition will not always be used.

\subsection{Approximation complexes}

We first identify a necessary condition for the integrality of a symmetric algebra $S(E)$; it is a strengthening of the condition $\left({ }^{*}\right)$. More generally define $\mathcal{F}_{k}$ as:

$$
\operatorname{height}\left(I_{t}(\varphi)\right) \geq \operatorname{rank}(\varphi)-t+1+k, \quad 1 \leq t \leq \operatorname{rank}(\varphi) .
$$

If $R$ is catenarian and $S(E)$ is an integral domain, pick a nonzero element $x$ lying in all the $I_{t}(\varphi)$ 's and such that $\operatorname{rank}_{R}(E)=\operatorname{rank}_{R /(x)}(E / x E)$. From the theorem above it follows that $E$ must satisfy $\mathcal{F}_{1}$.

One can rephrase $\mathcal{F}_{1}$ in terms of how $L$, the kernel of $\varphi$, embeds into $R^{n}$. It means that for each prime ideal $P$ where the localization $E_{P}$ is not a free module then

$$
v\left(E_{P}\right) \leq \operatorname{rank}(E)+\operatorname{height}(P)-1 .
$$

This says that for each prime ideal $P$ of $R, L_{P}$ decomposes into a summand of $R_{P}^{n}$ and a submodule $K$ of rank at most height $(P)-1$ (cf: [4], [13]).

We examine the impact of this condition on modules of projective dimension two. There are but a few instances where this has been examined in detail. Let

$$
0 \longrightarrow R^{p} \stackrel{\psi}{\longrightarrow} R^{m} \stackrel{\varphi}{\longrightarrow} R^{n} \longrightarrow E \longrightarrow 0
$$

be a free resolution of the module $E$.

One of the difficulties to be faced is the following. For $S(E)$ to be a domain the symmetric powers $S_{t}(E)$ must be torsion-free modules. If, from the presentation above, one could derive free resolutions for the $S_{t}(E)$ 
the hurdle largely would disappear. We could try, for instance, to use the complexes of [17]. For $t=2$ one has the complex $C$ :

$$
0 \rightarrow D_{2}\left(R^{p}\right) \rightarrow R^{p} \otimes R^{m} \rightarrow R^{p} \otimes R^{n} \oplus \wedge^{2} R^{m} \rightarrow R^{m} \otimes R^{n} \rightarrow S_{2}\left(R^{n}\right) \rightarrow 0 .
$$

For $\mathcal{C}$ to be acyclic-in the presence of $\mathcal{F}_{1}$-requires height $\left(I_{p}(\psi)\right) \geq 4$, cf. [17]. Thus if in addition $E$ is a reflexive module, it will be satisfied. Consequently we obtain:

Proposition 2.1.1 Let $R$ be a four-dimensional domain and let $E$ be a reflexive module of projective dimension two. Then $S(E)$ is not an integral domain.

The alternative approach has been to replace free complexes over the symmetric powers of $E$ by complexes of modules with sufficiently high depth to permit the use of the lemme d'acyclicité. One of the more direct ways is the following modified version of the Koszul complex. Assume that $R$ is a normal domain and $E$ is a torsion free $R$-module. To the presentation of $E$

$$
0 \longrightarrow L \longrightarrow R^{n} \longrightarrow E \longrightarrow 0
$$

we can associate the Koszul complex $\mathcal{K}$ whose component in degree $j$ is $K_{j}=$ $\wedge^{j}(L) \otimes S_{t-j}\left(R^{n}\right) . \mathcal{K}$ behaves much like the ordinary Koszul complex, in particular if a submodule of $L$ splits off $R^{n}$ then the corresponding complex is homotopic to $\mathcal{K}$. The drawback is that the exterior powers of $L$ may not have sufficiently high depth-at least for higher values of $t$. There are two modifications of $\mathcal{K}$ that have been proved useful: To mod out the $R$-torsion of $\mathcal{K}$-a step that cuts the length of the complex--or, more drastically, replace each $K_{j}$ by its bi-dual. They both preserve the 0 -dimensional homology$S_{t}(E)$-and the mapping cone property mentioned above (see [5] for details).

\subsection{Codimension two}

Perhaps the simplest interesting case is when $\operatorname{rank}(L)=2$. We then have the complex:

$$
0 \rightarrow\left(\wedge^{2} L\right)^{* *} \otimes S_{t-2}\left(R^{n}\right) \rightarrow L \otimes S_{t-1}\left(R^{n}\right) \rightarrow S_{t}\left(R^{n}\right) \rightarrow S_{t}(E) \rightarrow 0 .
$$

If $E$ has projective dimension finite, not necessarily two-or, if $R$ is a factorial domain $-\left(\wedge^{2} L\right)^{* *} \cong R$. It follows that if $E$ satisfies $\mathcal{F}_{1}$ then $S(E)$ is a domain. In fact, if $R$ is a Cohen-Macaulay ring, then $S(E)$ is a codimension 
two Cohen-Macaulay domain if and only if $L$ has projective dimension at most one (cf. [13]).

This short complex typifies some of the differences between the approximation complexes and projective resolutions of symmetric powers.

\subsection{Codimension three}

Consider a module $E$ satisfying $\mathcal{F}_{1}$. For a resolution of $E$, denote $L=$ $\operatorname{ker}(\varphi)$. The approximation complex leading to the symmetric square of $E$ is:

$$
0 \longrightarrow\left(\wedge^{2} L\right)^{* *} \longrightarrow L \otimes R^{n} \longrightarrow S_{2}\left(R^{n}\right) \longrightarrow S_{2}(E) \longrightarrow 0 .
$$

On account of $\mathcal{F}_{1}$, it follows that $S_{2}(E)$ will be torsion free if and only if the module $\left(\wedge^{2} L\right)^{* *}$ has depth at least 3 . (We may assume that the dimension of $R$ is at least 4.)

Let us assume that $J(E)$ has codimension three, that is, $L$ has rank three. The full approximation complex for $S(E)$ is easily obtained. The following notation is used: $B$ for the polynomial ring $R\left[T_{1}, \ldots, T_{n}\right]$ graded in the $T$ 's variables in the usual manner, and $B(-d)$ for the module isomorphic to $B$ but with the grading shifted by $d$ degrees to the left. The components of the approximation complex $Z(E)$ are put together from

$$
\left(\wedge^{t} L\right)^{* *}= \begin{cases}L^{*}, & t=2 \\ R, & t=3\end{cases}
$$

$0 \longrightarrow B(-3) \stackrel{\lambda}{\longrightarrow} L^{*} \otimes B(-2) \longrightarrow L \otimes B(-1) \longrightarrow B \longrightarrow S(E) \longrightarrow 0$.

It follows readily from [5] that if $E$ satisfies $\mathcal{F}_{1}$ then $Z(E)$ is acyclic.

We consider two cases where these considerations fit.

Theorem 2.3.1 Let $R$ be a regular local ring containing a field, of dimension at least four. Let $E$ be a reflexive module, free on the punctured spectrum of $R$ and such that $S(E)$ is an integral domain of codimension three. Then $E$ is a free module, that is $S(E)$ is a polynomial ring over $R$.

Proof. We plug all these conditions into the approximation complex $Z(E)$.

Since $E$ is a reflexive module, it follows that $L$ is a third-syzygy module. On the other hand, the degree two component of $Z(E)$ is 


$$
0 \rightarrow\left(\wedge^{2} L\right)^{* *} \rightarrow L \otimes B_{1} \rightarrow B_{2} \rightarrow S_{2}(E) \rightarrow 0,
$$

and as $S_{2}(E)$ is torsion-free, $\left(\wedge^{2} L\right)^{* *} \simeq L^{*}$ is also a third-syzygy module. It follows that $G=\operatorname{coker}(\lambda)$ is a rank $2 B$-module readily seen to have Serre's condition $S_{3}$. By the syzygy theorem of [2], $G$ is $B$-free and consequently $L^{*} \otimes B$ is also $B$-free. Therefore $L$ is $R$-free, that is $S(E)$ iss a complete intersection of codimension three. Thus $Z(E)$ is a minimal resolution of $S(E)$, so that $G$ cannot be $B$-free.

For the next result emphasis will be placed on a torsion-free module $E$ of projective dimension two, whose bi-dual $E^{* *}$ is a free module. This permits connecting the properties of $S(E)$ to those of the Cohen-Macaulay module $C$ :

$$
0 \longrightarrow E \longrightarrow R^{e} \longrightarrow C \longrightarrow 0 .
$$

This restriction on $E$ is aimed at emcompassing the already extensive theory when $E$ is an ideal. To get the missing coefficient of $Z(E)$, dualize the sequence

$$
0 \longrightarrow L \longrightarrow R^{n} \longrightarrow E \longrightarrow 0
$$

to get

$$
0 \rightarrow E^{*} \simeq\left(R^{e}\right)^{*} \rightarrow\left(R^{n}\right)^{*} \rightarrow L^{*} \rightarrow E_{x t}(E, R) \simeq E x t^{2}(C, R)=0,
$$

so that $L^{*}$ has projective dimension one.

Theorem 2.3.2 Let $E$ be a module as above satisfying $\mathcal{F}_{1}$. Then:

1. The complex $Z(E)$ is acyclic and $S(E)$ is a Cohen-Macaulay integral domain.

2. If $R$ is factorial then every ideal of $R$ of height at least 5 contains a prime element of $S(E)$.

Proof. The first assertion follows directly from [5, Theorem 6.2] once the depths of $L$ and $L^{*}$ are taken into account. Suppose $P$ is a nonzero prime of $R$ associated to $S(E)$; localizing at $P$ we may assume that $P$ is the maximal ideal of $R$. If $\operatorname{dim} R \leq 3, S(E)$ is actually of codimension two and [13, Theorem 3.5] applies. Finally, if $\operatorname{dim} R \geq 4$, the complex above says that each component $S_{t}(E)$ has depth at least $\operatorname{dim} R-3$. 
Let $x$ be a prime element of $R$ outside of any associated prime of $C$. Reduction mod $(x)$ has the following effects. (i) $E / x E$ is a torsion free module over $R /(x)$ and $L / x L$ is the first module of syzygies of $E / x E$. (ii) Because $L^{*}$ is the second module of syzygies of $W=E x t^{3}(C, R)$ and $x$ is regular on $W$ as well, it follows that $L^{*} / x L^{*}=(L / x L)^{*}$. (iii) To 'cycle the theorem' we must show that a prime ideal $P$ of $R$ of height at least five contains a prime element $x$ such that $\mathcal{F}_{1}$ holds for the module $E / x E$. This can be expressed as follows:

$$
\text { height }\left(I_{t}(\varphi), x\right) \geq \begin{cases}5 & t=1 \\ 4 & t=2 \\ 3 & t=3\end{cases}
$$

The existence of such element $\boldsymbol{x}$ is clear.

A consequence of this formulation is that the Theorem applies to the module image $\left(\psi^{*}\right)$ as well: It uses exactly the same hypotheses as the module $E$.

From [4, Theorem 5.8], one can put together numerical information about a projective resolution of $S(E)$ as an $B$-module:

$0 \rightarrow B(-3) \oplus B(-2)^{e} \rightarrow B(-2)^{n} \oplus B(-1)^{p} \rightarrow B(-1)^{m} \rightarrow B \rightarrow S(E) \rightarrow 0$.

If $R$ is a factorial domain, and $S(E)$ is integrally closed, we describe the divisorial prime ideals of $S(E)$ and its divisor class group.

If $P$ is a height one prime of $S(E)$, and $P \cap R=\mathbf{p} \neq(0)$, then $P=T(\mathbf{p})$. By the theorem above we must have that if $P$ is not contained in one of the minimal primes of $V\left(I_{1}(\varphi)\right)$ then it is principal.

The possible heights of the ideal $\mathbf{p}$ are: $0,1,3$ and 4 . If $\mathbf{p}=(0)$, since the localization $S(E)_{O}$ is a polynomial ring one can shift the support of $P$ to an isomorphic ideal $Q$ that has a nonzero contraction with $R$. If height $(\mathbf{p})=1, P=T(\mathbf{p})$, with $\mathbf{p}=x R$; this time consider the ideal $x P^{-1}$ to obtain an ideal in the same class as $P^{-1}$ whose contraction to $R$ has height at least two.

This leaves as generators for the divisor class group of $S(E), C l(S(E))$, the classes of $T(\mathbf{p})$, where $\mathbf{p}$ is a prime in $A s s(C)$, and of $T(\mathbf{q})$, with $\mathbf{q}$ a prime of height four containing $I_{1}(\varphi)$. To show these classes are linearly independent we use an argument of [12], [16]. Suppose there is a relation $([I]$ denotes the divisorial class of the ideal $I$ in $C l(S(E)))$ :

$$
\sum_{i} r_{i}\left[T\left(\mathbf{p}_{i}\right)\right]+\sum_{j} s_{j}\left[T\left(\mathbf{q}_{j}\right)\right]=0
$$


Localizing at each of the $\mathbf{p}_{i}$ gives that $r_{i}=0$, since obviously the image of $T\left(\mathbf{p}_{i}\right)$ cannot be a principal ideal. Then moving over to the $\mathbf{q}_{j}$ show that $s_{j}=0$ as well.

Denoting by $r$ the cardinality of the set of associated primes of $C$, and by $s$ that of the set of primes of height four that contain $I_{1}(\varphi)$, this proves:

Theorem 2.3.3 Let $R$ be a factorial domain and let $E$ be an $R$-module as above. If $S(E)$ is integrally closed, its divisor class group is a free abelian group of rank $r+s$.

Remark 2.3.4 To decide whether $S(E)$ is normal is dealt with by the method of [14]. It is simplified by the fact that the prime ideals that must be tested for have been identified: For each of the prime ideals of height 3 or 4 singled out above, the module

$$
(J(E)+P B) /\left(J(E)+P^{2} B\right),
$$

must have rank one as an $B / P B$-module; [14] describes ways of setting up the computation.

Remark 2.3.5 The proof of this theorem is very suggestive of the location of the generators of the divisor class group of a normal symmetric algebra.

\subsection{Codimension four}

Let $R$ be a Gorenstein domain and let $E$ be a module of projective dimension two, satisfying $\mathcal{F}_{1}$; assume $\operatorname{rank}(L)=4$. The coefficient modules of the complex $Z(E)$ are

$$
L_{t}=\left(\wedge^{t} L\right)^{* *}, 1 \leq t \leq 4 .
$$

Observe that $L_{3}=L^{*}$ and that $L_{2}$ is self-dual.

It follows easily that:

1. If $E$ is as in 2.3.2, then $Z(E)$ is acyclic. Without those conditions it is not possible to predict the depth of $L^{*}$ and $Z(E)$ may fail to be acyclic.

2. $S(E)$ is an integral domain if and only if depth $L_{2} \geq 3$.

3. $S(E)$ is Cohen-Macaulay if and only if $\operatorname{depth} L_{2} \geq \operatorname{dim} R-2$. 
The first two assertions follow because of the underlying mapping cone construction of $Z(E)$, as used in Theorem 2.3.2, while the last comes from $[5$, Theorem 6.2].

Example 2.4.1 Let us consider one example indicative of the method. Let $X$ and $Y$ be two generic, $4 \times 4$, skew-symmetric matrices in the variables $\left\{x_{i j}\right\}$ and $\left\{y_{i j}\right\}$ respectively. As in Example 1.1.8, let $I$ be the ideal defined by the entries of $[X, Y]$. It is generated by 6 elements; its jacobian matrix with respect to the $y_{i j}$-variables is:

$$
\varphi=\left[\begin{array}{rrrrrr}
0 & -x_{4} & -x_{5} & x_{2} & x_{3} & 0 \\
x_{4} & 0 & -x_{6} & -x_{1} & 0 & x_{3} \\
x_{5} & x_{6} & 0 & 0 & -x_{1} & -x_{2} \\
-x_{2} & x_{1} & 0 & 0 & -x_{6} & x_{5} \\
-x_{3} & 0 & x_{1} & x_{6} & 0 & -x_{4} \\
0 & -x_{3} & x_{2} & -x_{5} & x_{4} & 0
\end{array}\right] .
$$

The module $E_{4}=\operatorname{coker}(\varphi)$ satisfies $\mathcal{F}_{1}$ and has projective dimension two. Its first order syzygies $L=\operatorname{image}(\varphi)$ has for presentation matrix

$$
\psi=\left[\begin{array}{rr}
x_{1} & x_{6} \\
x_{2} & -x_{5} \\
x_{3} & x_{4} \\
x_{4} & x_{3} \\
x_{5} & -x_{2} \\
x_{6} & x_{1}
\end{array}\right]
$$

A calculation using Macaulay shows that $L \simeq L^{*}$, which are then of depth 5. A bit more of work shows that depth $L_{2}=4$; it mainly appeals to the fact that $L_{2}$ is self-dual: A presentation for $\wedge^{2} L$ can be obtained from $\psi$ and therefore $L_{2}$ is the first order syzygies of its dual. From the remarks above it follows that $S(E)$ is a Cohen-Macaulay integral domain.

It is clear that in codimension four the theory of the approximation complexes is not equal to the task of deciding whether a symmetric algebra $S(E)$ is an integral domain. The "corrections" to be introduced have yet to be identified.

We now seek conditions under which the conditions 2 and 3 above may occur in the setting of Theorem 2.3.2, but in codimension four. The module

$$
W=E x t^{2}(E, R) \simeq E x t^{3}(C, R),
$$


will play the role of a canonical module.

Proposition 2.4.2 The following are equivalent:

1. depth $L_{2} \geq \operatorname{dim} R-1$.

2. $S_{2}(W)$ is a Cohen-Macaulay module.

Proof. We follow [15] where a version of this result appears. Consider the canonical embedding of $\wedge^{2} L$ into $L_{2}$ :

$$
0 \longrightarrow \wedge^{2} L \longrightarrow L_{2} \longrightarrow D \longrightarrow 0 .
$$

Note that $D$ is a module of codimension three. On the other hand, the minimal resolution of $\wedge^{2} L$ is:

$$
0 \longrightarrow D_{2}\left(R^{p}\right) \longrightarrow R^{p} \otimes R^{m} \longrightarrow \wedge^{2} R^{m} \longrightarrow \wedge^{2} L \longrightarrow 0 .
$$

It follows that if depth $L_{2} \geq \operatorname{dim} R-1, D$ is a Cohen-Macaulay module. The long exact sequence of the functor $\operatorname{Hom}(-, R)$ yields:

$$
0 \rightarrow E x t^{2}\left(L_{2}, R\right) \rightarrow E x t^{2}\left(\wedge^{2} L, R\right) \rightarrow E x t^{3}(D, R) \rightarrow E x t^{3}\left(L_{2}, R\right) \rightarrow 0 .
$$

Since the end modules vanish and $E x t^{3}\left(\wedge^{2} L, R\right)=S_{2}(W)$, we get that $S_{2}(W)$ is also Cohen-Macaulay.

To obtain the converse, we must show that

$$
\operatorname{Ext}^{i}\left(L_{2}, R\right)=0, \text { for } i \geq 2 .
$$

First, by localizing at a prime in $A s s(W)$ we establish it for $i=2$. At a prime of height $4, L$ decomposes into a rank 3 module $M$ and a free summand. This will imply that $L_{2} \cong M \oplus M^{*}$, and therefore has the desired depth. This takes care of the case $i=3$. Finally, observe that in all cases $D=\operatorname{Ext}^{3}\left(S_{2}(W), R\right)$.

\subsection{Computational remarks}

Let $R$ be a polynomial ring over a prime field, and let $E$ be a finitely generated $R$-module. Deciding whether the defining ideal $J(E)$ of $S(E)$ is prime, in an environment provided by programs such as Macaulay, Macsyma or Reduce, at the most primitive level, involves finding an element $f \in R$ 
for which $E_{f}$ is a free module over the localization $R_{f}$ and checking for $J(E): f=J(E)$.

This element may be found in the determinantal ideal that defines the free locus of $E$, obtainable from a presentation of $E$. It often turns out to be too "long" to be practical. An alternative is to go further down in the resolution of $E$. For instance, suppose it is known that $E$ satisfies $\mathcal{F}_{1}$. Pick $f$ such that for the module $L$ of first-order syzygies of $E$, the localization $L_{f}$ is a free module. It will follow that $S(E)$ is a domain if the equation above holds. Its main advantage is to broaden the choice of the $f$ 's into more amenable polynomials.

As for studying the Cohen-Macaulayness of $J(E)$, a suitable approach has been to determine regular systems of parameters. The attempt to go directly for their projective dimension is usually defeated if the number of generators - and therefore the number of the $T_{i}$-variables -is large.

\section{References}

[1] L. Avramov, Complete intersections and symmetric algebras, J. Algebra 73 (1980), 249-280

[2] E. G. Evans and P. Griffith, The syzygy problem, Annals of Math. 114 (1981), 323-333

[3] O. Forster, Über die Anzahl der Erzeugenden eines Ideals in einem Noetherschen Ring, Math. Z. 84 (1964), 80-87

[4] J. Herzog, A. Simis and W. V. Vasconcelos, Koszul homology and blowing-up rings, Proc. Trento Commutative Algebra Conf., Lectures Notes in Pure and Applied Math., vol. 84, Dekker, New York, 1983, $79-169$

[5] J. Herzog, A. Simis and W. V. Vasconcelos, On the arithmetic and homology of algebras of linear type, Trans. Amer. Math. Soc. 283 (1984), $661-683$

[6] K. Hulek, A remark on certain matrix varieties, Linear and Multilinear Algebra 10 (1981), 169-172

[7] C. Huneke, On the symmetric algebra of a module, J. Algebra 69 (1981), 113-119 
[8] C. Huneke and M. E. Rossi, The dimension and components of symmetric algebras, J. Algebra 98 (1986), 200-210

[9] T. Jòsefiak, Ideals generated by the minors of a symmetric matrix, Comment. Math. Helvetici 53 (1978), 595-607

[10] H. Matsumura, Commutative Ring Theory, Cambridge University Press, 1986

[11] T. Motzkin and O. Taussky, Pairs of matrices with property L II, Trans. Amer. Math. Soc. 80 (1955), 387-401

[12] A. Simis and N. V. Trung, Divisor class group of ordinary and symbolic blow-ups, Math. Z., to appear

[13] A. Simis and W. V. Vasconcelos, On the dimension and integrality of symmetric algebras, Math. Z. 177 (1981), 341-358

[14] W. V. Vasconcelos, On linear complete intersections, J. Algebra, to appear

[15] W. V. Vasconcelos, Koszul homology and the structure of low codimension Cohen-Macaulay ideals, Trans. Amer. Math. Soc. 301 (1987), $591-613$

[16] R. Villarreal, Koszul Homology of Cohen-Macaulay Ideals, Ph. D. Thesis, Rutgers University, 1986

[17] J. Weyman, Resolutions of the exterior and symmetric powers of a module, J. Algebra 58 (1979), 333-341

Instituto de Matemática

Universidade Federal da Bahia

40160 Salvador, Bahia, Brasil

Department of Mathematics

Rutgers University

New Brunswick, New Jersey 08903, USA 